\title{
Detecting Beef Tallow adulteration in Palm Oil with NIR Spectroscopy and Chemometrics Analysis
}

\author{
Mohd Fared Abdul Khir ${ }^{1 *}$, Nur Zahidah Syahirah Marzuki ${ }^{2}$, Nur Azera \\ Tuhaime $^{2}$, Katrul Nadia Basri ${ }^{2,3}$ and Mutia Nurulhusna Hussain ${ }^{2,4}$
}

\author{
${ }^{1}$ Faculty of Science and technology, Universiti Sains Islam (USIM), 71800 Nilai, Negeri Sembilan, MALAYSIA \\ ${ }^{2}$ Photonics R\&D, Mimos Berhad, Technology Park Malaysia, 57000 Kuala Lumpur, MALAYSIA \\ ${ }^{3}$ Center of Engineering, Universiti Teknologi MARA (UiTM), 40450 Shah Alam, Selangor, MALAYSIA \\ ${ }^{4}$ Institut Penyelidikan Produk Halal, Universiti Putra Malaysia (UPM), 43400 Serdang, Selangor, MALAYSIA
}

Received 4 February 2018; accepted 10 April 2018, available online 30 April 2018

\begin{abstract}
The NIR spectroscopy combined with chemometrics analysis has started to gain interest in the food industry for its rapid and non-destructive advantage. We report our result in investigating the capability of the NIR spectroscopy and chemometrics analysis in classifying pure palm oil samples from the one adulterated with beef tallow. The result analyzed using the open source $\mathrm{R}$ software has shown that the adulterated palm oil samples with adulteration of $0.5 \%$ and above were correctly classified
\end{abstract}

Keywords: NIR spectroscopy, Chemometrics, R. Adulteration, Palm Oil

\section{Introduction}

Recently, the Near Infrared (NIR) spectroscopy combined with the chemometrics analysis or also known as the multivariate analysis have started to become an important tool in the food industry. While known for its rapid and non-destructive measurement capability [1-3], the recent advancement in miniaturized type NIR spectrometer gives users portability advantage, making it suitable for on-site measurement. It is usually less expensive and less bulky although one may argue from the point of accuracy performance as compared to the Fourrier-transform infrared (FTIR) spectroscopy or even the Raman spectroscopy. In NIR spectroscopy, the radiation within near infrared range $(700 \mathrm{~nm} \sim 2500 \mathrm{~nm})$ is absorbed by the sample. The difference in absorption at every wavelength forms a pattern in the output spectrum which consists of the overtones or their combinations. The output spectrum is then subject to further statistical analysis such as the chemometrics analysis. The absorption concept in NIR spectroscopy can be simply explained by the Beer Lambert Law as follows.

$A=\varepsilon c l$

where $A$ is the absorption, $\varepsilon$ is the absorptivity, $c$ is the concentration of molecules and $l$ is the optical path length

The NIR spectroscopy is known to be widely used for quantification analysis [4]. However, many recent works related to qualification analysis with NIR spectroscopy were reported. Studies in food authentication utilizing NIR spectroscopy can be found for example in [5-8]. Classification of pure olive oil from the one adulterated with sunflower oil can be found in [5]. The classification of pure palm oil and the one adulterated with chicken fat can be seen in [6]. These works have shown good result with over $90 \%$ correct classification accuracy. It is not difficult to explain the reason for this as the oils and fats differ in their fatty acid composition [4]. The different in the degree of unsaturation will be reflected in the NIR spectrum and can be simply analyzed for classification using chemometrics analysis [4]. As for the case of detecting beef tallow adulteration in palm oil, we have not found a literature that relates to the NIR spectroscopy and chemometrics analysis.

In this work, we have conducted an experiment to investigate the feasibility of classifying the pure palm oil and the one adulterated with beef tallow. A longwave NIR spectrometer was used to obtain the NIR spectrum which was then analyzed using an open source $\mathrm{R}$ software for classification and also quantification [9]. The next section describes the experimental setup. Section three discusses the result. Section four conclude and suggests future works.

\section{Materials and experiment setup}

The palm oil and the beef tallow were obtained from local market. The adulterated sample set was constructed by mixing the pure palm oil and beef tallow at concentration ranges of $0.5 \% \sim 50 \%(\mathrm{v} / \mathrm{v})$ and were labeled according to the percentage of the beef tallow in palm oil. Depending on the nature of the sample to be measured, one can either use reflection, transmission or transflectance type of NIR measurement. In this 
experiment, as the sample is of liquid type, we used the transmission setup as depicted in Figure 1. The all in one spectrometer is an off-the-shelf setup that comes together with the light source and a small compartment for cuvette holder to place the sample. The spectrometer is the DLPNIRScanEVM from Texas Instrument. which utilizes digital light processing (DLP) technology [10]. We made some amendments by preparing the body cover and the top cover of the cuvette holder compartment as an effort to reduce background noise. Each measurement gives intensity at 275 wavelengths ranging from $1350 \mathrm{~nm}$ until $2450 \mathrm{~nm}$ with $4 \mathrm{~nm}$ interval.

Each sample was first heated to $60^{\circ} \mathrm{C}$ and rotated using a magnetic stirrer to ensure its homogeneity before being placed inside a $1 \mathrm{~cm}$ square cuvette. For each measurement, the absorbance value was calculated by subtracting a new background of air and empty cuvette. Each spectral data is an average measurement of 3 rounds.

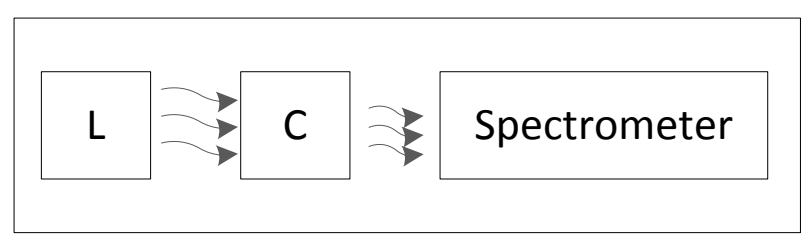

Figure 1 Schematic of the experimental setup. L;Polychromatic light source, C; Cuvette for sample holder, Spectrometer; the DLPNIRScanEVM spectrometer in use.

\section{Results and Discussion}

The combined spectrum of all samples is depicted in Figure 2. It includes the pure palm and the beef tallow adulterated palm. The spectrums look very much similar. Unlike with FTIR spectrums, the NIR spectrums are hardly distinguishable with bare eyes and need to rely on statistical method for analysis. As we have multi variables corresponding to the intensity reading at each of the 275 wavelengths, we can always rely on the wellknown chemometrics analysis for either qualification or quantification exercise. The region of interest in the case of oil and fat are the two regions at $1666 \mathrm{~nm} \sim 1818 \mathrm{~nm}$ and at the $2083 \mathrm{~nm} \sim 2222 \mathrm{~nm}$ (Yang et. al. 2005). The former corresponds to the first overtones of the $\mathrm{C}-\mathrm{H}$ stretching from $-\mathrm{CH} 2,-\mathrm{CH}$ and $-\mathrm{CH}=\mathrm{CH}$ - functional groups while the later corresponds to the combination of $\mathrm{C}-\mathrm{H}$ stretching related to cis double bonds and increases with the increase in the degree of unsaturation. One can expect differences at these regions of spectrums when the palm oil is adulterated with beef tallow as the degree of unsaturation will reduce with the increase in the beef tallow percentage in the mixture.

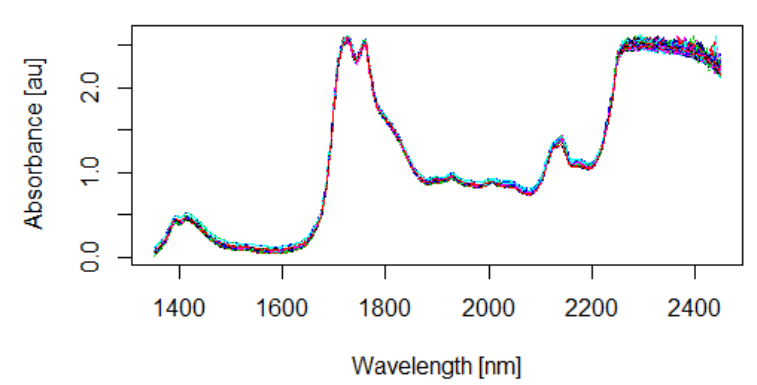

Figure 2 The raw absorbance spectral of all samples

We have made use of the $\mathrm{R}$ open source software for the multi-variate analysis, specifically the caret package [11-12] and the pls package in [13] The spectral data set was first analyzed for qualification to investigate if the palm and the beef tallow adulterated palm can be classified to their correct group i.e. the pure and the adulterated group. In short, it is a two class problem. Two classifiers i.e. the Linear Discriminant Analysis (LDA) and the Partial Least Square (PLS) were used to produce the calibration model and compared their performance. All spectrums were normalized and centered before applying the said two classifiers with leave one out (LOO) cross validation. The result is depicted in Table 1.

\begin{tabular}{llllll}
\hline \hline \multicolumn{6}{c}{ Number of principle components } \\
\hline \multicolumn{7}{c}{1} & 2 & 3 & 4 & 5 \\
\hline \multicolumn{2}{c}{ Accuracy } & & & & \\
\hline LDA & 0.9783 & 0.9783 & 0.9783 & 0.9783 & 0.9783 \\
\hline PLS & 0.8913 & 0.9783 & 1 & 1 & 1 \\
\hline Sensitivity & & & & \\
\hline LDA & 1 & 1 & 1 & 1 & 1 \\
\hline PLS & 0.9762 & 1 & 1 & 1 & 1 \\
\hline \hline
\end{tabular}

Table 1 The accuracy and sensitivity by the number of principle components used by each model

It is common to use the score plot to visualize the classification results. However, they do not really represent the final classification result as one may end up with only two principle components in a 2-D plot or at most three principle components in a 3-D plot. Hence in this article, we prefer the use of the confusion matrix which is a direct representation of the prediction result, although seems less graphical. The simplest metric for classification evaluation is the overall accuracy rate. It reflects the agreement between the observed and predicted classes and has the most straight forward interpretation [14]. The overall accuracy can be derived from the confusion matrix. For example, the following Figure 3 shows the confusion matrix generated by $\mathrm{R}$ for the case of the .PLS model when two principle components were used. Since the correctly classified samples are 45 from the total 46 samples, the overall 
classification accuracy is 0.9783 . The sensitivity is 1 as all adulterated samples were correctly detected as adulterated.

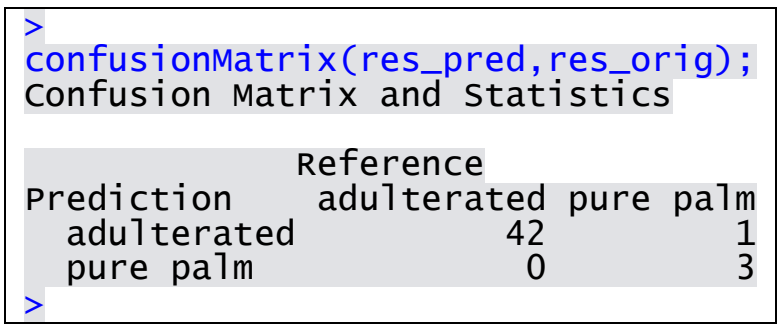

Figure 3 Confusion matrix for the case of PLS model when two principle components were used.

In general, both models give good result, close to $100 \%$ overall classification accuracy. The PLS result shows that all samples were correctly classified when three or more principle components were in use while the LDA did not manage to have all correct classification even if five principle components were used. One can choose to have many more principle components for example ten or even twenty but there is always a concern on the problem of overfitting. If a model is over fitted, one may obtain a good prediction result only for the data set internal to the calibration model but not for data sets external to it, i.e. affecting the robustness aspect of the calibration model.

Apart from the overall accuracy parameter, the one that we are interested in is the sensitivity parameter as we would like to know the capability of detecting the adulterated sample. As can be seen from Table 1, both models show high sensitivity close to 1 which means that both models were almost perfectly successful in detecting the adulterated samples. In the case of LDA, the sensitivity is 1 regardless of the number of principle component in use while for the case of PLS, the sensitivity reaches 1 starting from three principle components and above. From the results of overall accuracy and the sensitivity, we can say that the two models are good enough for the classification exercise.

Let us now turn to the quantification analysis. Using the PLS regression with leave one out (LOO) cross validated predictions, it was found that the correlation of determination $\left(\mathrm{R}^{2}\right)$ value started to show less improvement when four principle components are in use. As such, we used four principle components for the prediction plot depicted in Figure 5. To be more precise, the $\mathrm{R}^{2}$ value with four principle components is 0.9948 and can be considered good as an $\mathrm{R}^{2}>90 \%$ shows good prediction capability [15].

\section{Prediction Plot}

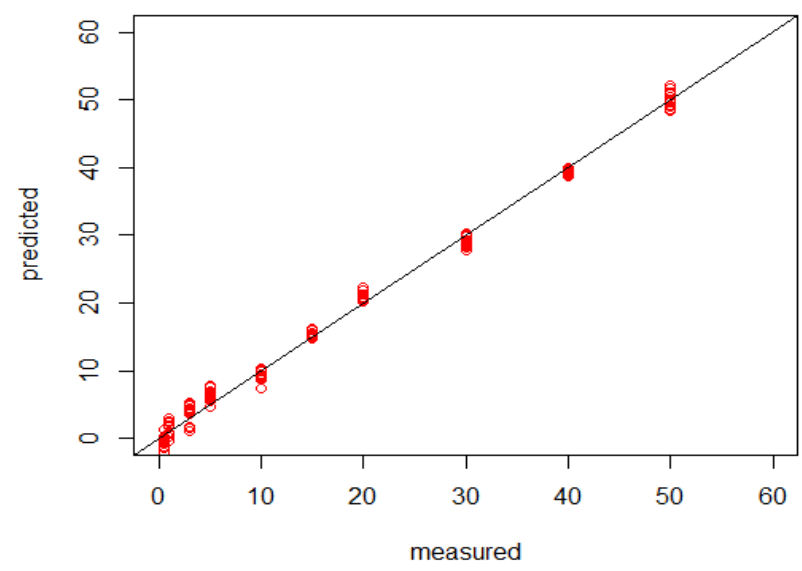

Figure 3 The prediction plot, modeled with PLS using 4 principle components.

\section{Conclusion}

It has been shown in this work that the longwave NIR spectroscopy combined with chemometrics analysis is capable of detecting beef tallow adulteration in palm oil with above $0.5 \%$ adulteration $(\mathrm{v} / \mathrm{v})$. An open source $\mathrm{R}$ software with a commonly used classifiers either the LDA or the PLS is enough to yield a good result. Other chemometrics algorithm can be used to improve the result

\section{References}

[1] Batten GD. 1998. Plant analysis using near infrared reflectance spectroscopy: the potential and the limitations. Aust. J. Expl. Agric.38: 697-706

[2] Williams P, Norris K. 2001. Near infrared technology in the agricultural and food industries, 2nd ed., American Association of Cereal Chemists Inc., St. Paul, MN, USA

[3] Bazar, G, Kover, G, Locsmandi, L, Szabo, A, Romvari, R. 2010. Detection of aliment adulteration by means of near infrared spectroscopy - a feasibility study based on open-source R project. Proceedings of the 14th International Conference on NIR Spectroscopy.

[4] Yang, H., Irudayaraj, J. \& Paradkar, M. M. 2005. Discriminant analysis of edible oils and fats by FTIR, FT-NIR and FT-Raman spectroscopy. Food Chemistry 93: 25-32.

[5] Downey, G., McIntyre, P. , Davies, A. N. (2002). Detection and quantifying sunflower oil adulteration in extra virgin olive oils from the eastern Mediterranean by visible and near-infrared spectroscopy. Journal of Agricultural and Food Chemistry. 50:5520-5525

[6] Abdul Khir, M. F., Hisham, M. H., Abdullah, M.S., Witjaksono, G. 2014. "Feasibility of Detecting Palm Oil Adulteration with Chicken Fat using NIR spectroscopy and Chemometrics Analysis". In 
Proceeding of the International Conference on Image Processing, Computers and Industrial Engineering (ICICIE2014), Kuala Lumpur, 2014.

[7] Hussain, M. N., Abdul Khir,M.F. Hisham, M. H., Yusof. Z. M. 2014. "Feasibilitystudyof Detecting Canola Oil Adulteration With Palm Oil Using NIR spectroscopy and Multivariate Analysis". In Proceeding of the International Conference on information \& Communication Technology and Systems(ICTS2014), Surabaya, 2014.

[8] Downey, G. \& Flynn, S. J.2002. Discrimination between virgin olive oils from Crete, the Peloponesse and other Greek Islands using nearinfrared transflectance spectroscopy. Near-infrared Spectroscopy Proceedings of the $10^{\text {th }}$ International Conference, Chichester: NIR Publications, pp 239241.

[9] "The $\mathrm{R}$ project for statistical computing". http://www.r-project.org. Accessed on $29^{\text {th }}$ December 2017.
[10] The DLP NIRScan Evaluation Module. http://www.ti.com/tool/dlpnirscanevm. Accessed on $29^{\text {th }}$ December 2017.

[11] The CARET package. http://topepo.github.io/caret/index.html. Accessed on $29^{\text {th }}$ December 2017.

[12] Kuhn M. 2008. Building Predictive models in $\mathrm{R}$ Using the caret package. Journal of Statistical Software. 28(5)

[13] Mevik, B., Wehrens, R. 2007. The pls Package: principle Component and Partial Least Squares regression in R. Journal of Statistical Software. $18(2)$

[14] Kuhn M. \& Johnson K. 2013. Applied Predictive Modelling. Springer

[15] Da Wen Sun. 2008. Modern Techniques for Food Authentication. Chapter 3: Spectroscopic Technique : Near Infrared (NIR) Spectroscopy. Academic Press.. 